STRUCTURE REPORTS

ISSN 1600-5368

Received 1 September 2014

Accepted 15 September 2014

Edited by M. Weil, Vienna University of Technology, Austria

Keywords: crystal structure; praseodymium ethylenediammonium; hydrogen bonds

CCDC reference: 1024418

Supporting information: this article has supporting information at journals.iucr.org/e

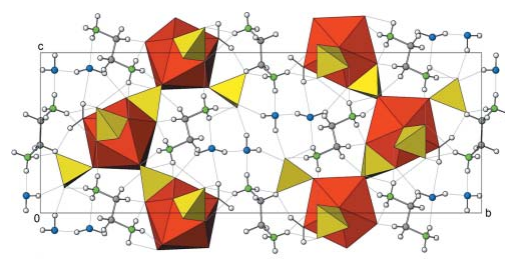

OPEN $\odot$ ACCESS

\section{Crystal structure of tris(ethylenediammonium) hexasulfatopraseodymium(III) hexahydrate}

\author{
Peter Held
}

Institut für Kristallographie, Universität zu Köln, Greinstrasse 6, D-50939 Köln, Germany. *Correspondence e-mail: peter.held@uni-koeln.de

In the title salt, $\left(\mathrm{C}_{2} \mathrm{H}_{10} \mathrm{~N}_{2}\right)_{3}\left[\mathrm{Pr}_{2}\left(\mathrm{SO}_{4}\right)_{6}\right] \cdot 6 \mathrm{H}_{2} \mathrm{O}$, the $\operatorname{Pr}^{\mathrm{III}}$ cation is surrounded ninefold by five sulfate groups (two monodentate and three chelating) and by one water molecule [range of $\mathrm{Pr}-\mathrm{O}$ bond lengths 2.383 (3) to 2.582 (3) A]. The $\left[\operatorname{Pr}\left(\mathrm{SO}_{4}\right)_{5}\left(\mathrm{H}_{2} \mathrm{O}\right)\right]$ groups are arranged in sheets parallel to (010). Two crystal water molecules and two ethylenediammonium cations (one with point group symmetry $\overline{1}$ ) connect the sheets via $\mathrm{O}-\mathrm{H} \cdots \mathrm{O}$ and $\mathrm{N}-\mathrm{H} \cdots \mathrm{O}$ hydrogen bonds from weak up to medium strength into a three-dimensional framework structure.

\section{Chemical context}

In the course of a systematic search for new 'double salts' of simple secondary amines and mono- or divalent cations of various inorganic acids, the structures of $\left(\mathrm{C}_{2} \mathrm{H}_{10} \mathrm{~N}_{2}\right)\left[\mathrm{Li}_{2}\left(\mathrm{SO}_{4}\right)_{2}\right]$ and $\left(\mathrm{C}_{2} \mathrm{H}_{8} \mathrm{~N}\right)\left[\mathrm{Cu}\left(\mathrm{HSO}_{4}\right)\left(\mathrm{SO}_{4}\right)\left(\mathrm{H}_{2} \mathrm{O}\right)_{4}\right]$ have been described previously (Held, 2003, 2014). In continuation of these studies, lithium was replaced by trivalent praseodymium, yielding crystals of the title compound with composition $\left(\mathrm{C}_{2} \mathrm{H}_{10} \mathrm{~N}_{2}\right)_{3}\left[\mathrm{Pr}_{2}\left(\mathrm{SO}_{4}\right)_{6}\right] \cdot 6 \mathrm{H}_{2} \mathrm{O}$.
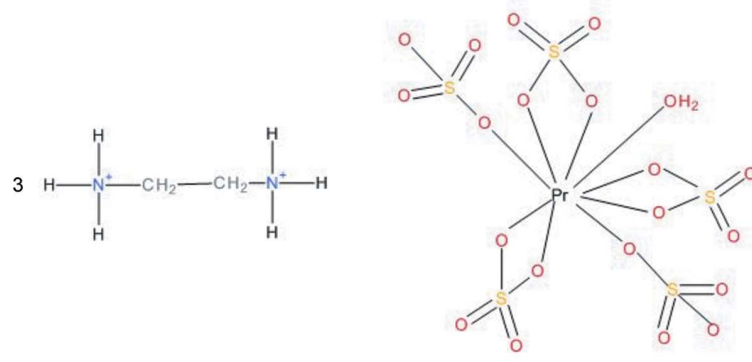

$6 \mathrm{H}_{2} \mathrm{O}$

\section{Structural commentary}

The asymmetric unit of the title compound contains three $\left(\mathrm{SO}_{4}\right)^{2-}$ anions, one and a half $\left[\mathrm{NH}_{2}\left(\mathrm{CH}_{3}\right)\right]^{2+}$ cations (the other half being generated by inversion symmetry), one $\mathrm{Pr}^{3+}$ cation as well as three water molecules (Fig. 1). The $\operatorname{Pr}^{3+}$ cation is surrounded by nine $\mathrm{O}$ atoms from five sulfate groups, two of which are monodentately bonding and three chelating, and of one water molecule. The averaged $\mathrm{Pr}-\mathrm{O}$ distance in the resulting distorted monocapped square-antiprism, $\left[\mathrm{Pr}\left(\mathrm{SO}_{4}\right)_{5}\left(\mathrm{H}_{2} \mathrm{O}\right)\right]$, is $2.52(7) \AA$. Praseodymium reaches an overall bond-valence sum (Brown \& Altermatt, 1985) of 3.23 valence units. The $\mathrm{S}-\mathrm{O}$ distances are nearly equal [average distance 1.479 (13) $\AA$ ], however, the $\mathrm{O}-\mathrm{S}-\mathrm{O}$ angles vary [average bond angle $109.48(2.05)^{\circ}$ ] clearly. One sulfate group (S2) interconnects two $\left[\mathrm{PrO}_{9}\right]$ polyhedra via two common edges parallel to [001], while another sulfate group (S3) 


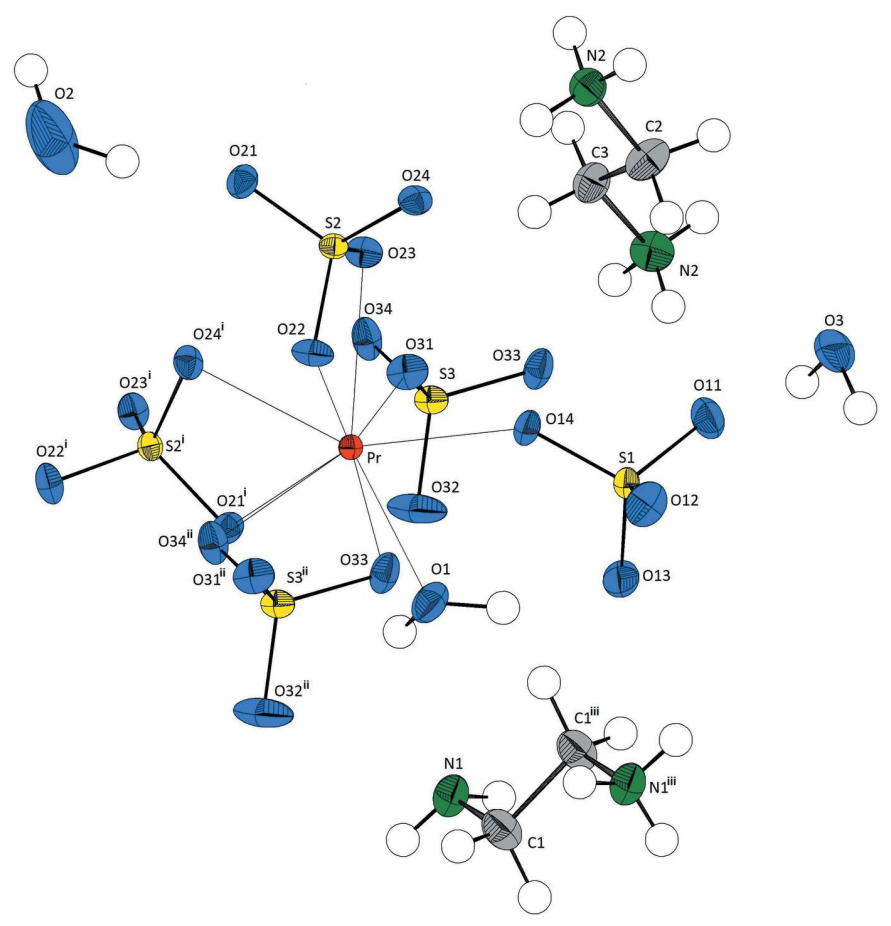

Figure 1

The molecular entities in the structure of the title compound, showing the atom-numbering scheme. Displacement ellipsoids are drawn at the $50 \%$ probability level. [Symmetry codes: (i) $x,-y+\frac{1}{2}, z+\frac{1}{2}$; (ii) $x+1, y, z$; (iii) $-x,-y,-z+1]$.

connects via a common edge and a common vertex parallel to [100], leading to the formation of sheets parallel to (010).

\section{Supramolecular features}

Hydrogen bonds of medium strength involving water molecules as donor groups and $\mathrm{O}$ atoms of the sulfate anions as acceptor groups interconnect neighbouring $\left[\mathrm{Pr}\left(\mathrm{SO}_{4}\right)_{5}\left(\mathrm{H}_{2} \mathrm{O}\right)\right]$ units. Together with relatively weaker $\mathrm{N}-\mathrm{H} \cdots \mathrm{O}$ hydrogen bonds of the ammonium groups atoms to sulfate anions, a three-dimensional framework is formed (Table 1, Fig. 2).

Table 1

Hydrogen-bond geometry $\left(\AA{ }^{\circ}\right)$.

\begin{tabular}{lllll}
\hline$D-\mathrm{H} \cdots A$ & $D-\mathrm{H}$ & $\mathrm{H} \cdots A$ & $D \cdots A$ & $D-\mathrm{H} \cdots A$ \\
\hline $\mathrm{O} 1-\mathrm{H} 11 \cdots \mathrm{O} 32$ & $0.72(8)$ & $2.53(8)$ & $2.974(6)$ & $121(7)$ \\
$\mathrm{O} 1-\mathrm{H} 12 \cdots \mathrm{O} 13$ & $0.78(6)$ & $1.92(6)$ & $2.674(5)$ & $162(6)$ \\
$\mathrm{O} 2-\mathrm{H} 21 \cdots \mathrm{O} 3$ & $1.00(12)$ & $1.89(12)$ & $2.858(7)$ & $163(9)$ \\
$\mathrm{O} 2-\mathrm{H} 22 \cdots \mathrm{O} 21^{\mathrm{i}}$ & $0.77(8)$ & $2.29(8)$ & $2.905(6)$ & $137(7)$ \\
$\mathrm{O} 3-\mathrm{H} 31 \cdots \mathrm{O} 11^{\mathrm{ii}}$ & $0.87(7)$ & $1.95(8)$ & $2.795(5)$ & $165(7)$ \\
$\mathrm{O} 3-\mathrm{H} 32 \cdots \mathrm{O} 12^{\mathrm{iii}}$ & $0.80(8)$ & $2.00(8)$ & $2.766(5)$ & $162(8)$ \\
$\mathrm{N} 1-\mathrm{H} 1 A \cdots \mathrm{O} 33$ & $0.87(8)$ & $2.48(8)$ & $3.291(5)$ & $155(6)$ \\
$\mathrm{N} 1-\mathrm{H} 1 B \cdots \mathrm{O} 3$ & $0.88(7)$ & $1.92(7)$ & $2.758(6)$ & $158(6)$ \\
$\mathrm{N} 1-\mathrm{H} 1 C \cdots \mathrm{O} 13^{\text {iv }}$ & $0.99(9)$ & $1.85(9)$ & $2.841(6)$ & $176(7)$ \\
$\mathrm{N} 2-\mathrm{H} 2 A \cdots \mathrm{O} 24$ & $0.76(7)$ & $2.21(7)$ & $2.976(5)$ & $177(7)$ \\
$\mathrm{N} 2-\mathrm{H} 2 B \cdots \mathrm{O} 22^{\mathrm{v}}$ & $0.83(8)$ & $2.17(8)$ & $2.967(6)$ & $162(7)$ \\
$\mathrm{N} 2-\mathrm{H} 2 C \cdots \mathrm{O} 34^{\text {vi }}$ & $0.94(7)$ & $2.20(6)$ & $3.020(5)$ & $146(5)$ \\
$\mathrm{N} 3-\mathrm{H} 3 A \cdots \mathrm{O} 2{ }^{\text {vii }}$ & $0.85(7)$ & $2.12(7)$ & $2.901(8)$ & $153(6)$ \\
$\mathrm{N} 3-\mathrm{H} 3 B \cdots \mathrm{O} 11$ & $0.90(7)$ & $1.95(8)$ & $2.847(6)$ & $175(6)$ \\
$\mathrm{N} 3-\mathrm{H} 3 C \cdots \mathrm{O} 33$ & $0.87(7)$ & $2.20(7)$ & $3.066(5)$ & $173(6)$ \\
\hline
\end{tabular}

Symmetry codes: (i) $x,-y+\frac{1}{2}, z+\frac{1}{2}$; (ii) $x, y, z+1$; (iii) $-x+1,-y,-z+1$; (iv) $-x,-y,-z+1$; (v) $x-1,-y+\frac{1}{2}, z-\frac{1}{2}$; (vi) $x, y, z-1$; (vii) $x-1, y, z-1$.

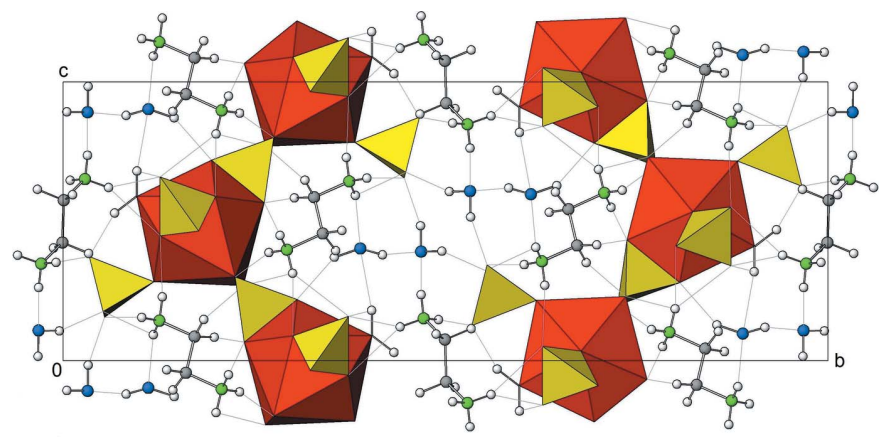

Figure 2

(100)-projection of the crystal structure of the title compound. Hydrogen bonds are shown as light-grey dashed lines. Colour scheme: $\left(\mathrm{SO}_{4}\right)$ tetrahedra (yellow), monocapped antiprism $\left[\mathrm{PrO}_{9}\right]$ (red), $\mathrm{O}$ (blue), $\mathrm{N}$ (green), C (grey), H (white).

\section{Synthesis and crystallization}

The title compound was obtained by reaction of an aqueous solution of praseodymium(III) sulfate with ethylenediamine and sulfuric acid $(18 \mathrm{~mol} / \mathrm{l})$ in a stoichiometric ratio 1:1:2. The title compound crystallized by slow evaporation of the solvent at room temperature in form of light-green crystals with dimensions up to $3 \mathrm{~mm}$ within a few weeks.

\section{Refinement}

Crystal data, data collection and structure refinement details are summarized in Table 2. All $\mathrm{H}$ atoms were clearly

Table 2

Experimental details.

Crystal data

Chemical formula

$M_{\mathrm{r}}$

Crystal system, space group

Temperature (K)

$a, b, c(\AA)$

$\beta\left({ }^{\circ}\right)$

$V\left(\AA^{3}\right)$

$Z$

Radiation type

$\mu\left(\mathrm{mm}^{-1}\right)$

Crystal size (mm)

Data collection

Diffractometer

Absorption correction

$T_{\min }, T_{\max }$

No. of measured, independent and observed $[I>2 \sigma(I)]$ reflections

$R_{\text {int }}$

$(\sin \theta / \lambda)_{\max }\left(\AA^{-1}\right)$

Refinement

$R\left[F^{2}>2 \sigma\left(F^{2}\right)\right], w R\left(F^{2}\right), S$

No. of reflections

No. of parameters

$\mathrm{H}$-atom treatment

$\Delta \rho_{\max }, \Delta \rho_{\min }\left(\mathrm{e} \AA^{-3}\right)$
$\left(\mathrm{C}_{2} \mathrm{H}_{10} \mathrm{~N}_{2}\right)_{3}\left[\mathrm{Pr}_{2}\left(\mathrm{SO}_{4}\right)_{6}\right] \cdot 6 \mathrm{H}_{2} \mathrm{O}$

1152.70

Monoclinic, $P 2_{1} / c$

295

6.6174 (8), 26.668 (4), 10.0264 (13)

$104.446(15)$

$1713.4(4)$

2

Mo $K \alpha$

3.29

$0.22 \times 0.21 \times 0.20$

Stoe IPDS-II

Multi-scan (X-SHAPE and $X$-RED32; Stoe \& Cie, 2002) $0.491,0.620$

$14346,3922,3091$

0.044

0.662

Computer programs: $X$-AREA (Stoe \& Cie, 2002), SIR92 (Altomare et al., 1993), SHELXL97 (Sheldrick, 2008), ATOMS (Dowty, 2002) and publCIF (Westrip, 2010). 
discernible from difference Fourier maps. Methylene $\mathrm{H}$ atoms were refined with a riding-model constraint, using a $\mathrm{C}-\mathrm{H}$ distance of $0.97 \AA$ and $U_{\text {iso }}(\mathrm{H})=1.2 U_{\text {eq }}(\mathrm{C})$. Ammonium and water $\mathrm{H}$ atoms were refined freely.

\section{Acknowledgements}

The author would like to thank Professor G. Meyer and Dr I. Pantenburg from the Institute of Inorganic Chemistry of the University of Cologne for the opportunity to collect data at the single-crystal diffractometer.

\section{References}

Altomare, A., Cascarano, G., Giacovazzo, C. \& Guagliardi, A. (1993). J. Appl. Cryst. 26, 343-350.

Brown, I. D. \& Altermatt, D. (1985). Acta Cryst. B41, 244-247.

Dowty, E. (2002). ATOMS. Shape Software, Kingsport, Tennessee, USA.

Held, P. (2003). Z. Kristallogr. New Cryst. Struct. 218, 13-14.

Held, P. (2014). Acta Cryst. E70, m119.

Sheldrick, G. M. (2008). Acta Cryst. A64, 112-122.

Stoe \& Cie (2002). $X$-AREA, X-RED32 and X-SHAPE. Stoe \& Cie, Darmstadt, Germany.

Westrip, S. P. (2010). J. Appl. Cryst. 43, 920-925. 


\section{supporting information}

Acta Cryst. (2014). E70, 235-237 [doi:10.1107/S1600536814020704]

\section{Crystal structure of tris(ethylenediammonium) hexasulfatopraseodymium(III) hexahydrate}

\section{Peter Held}

\section{Computing details}

Data collection: $X$-AREA (Stoe \& Cie, 2002); cell refinement: $X$-AREA (Stoe \& Cie, 2002); data reduction: $X$-AREA (Stoe \& Cie, 2002); program(s) used to solve structure: SIR92 (Altomare et al., 1993); program(s) used to refine structure: SHELXL97 (Sheldrick, 2008); molecular graphics: ATOMS (Dowty, 2002); software used to prepare material for publication: publCIF (Westrip, 2010).

\section{Tris(ethylenediammonium) hexasulfatopraseodymium(III) hexahydrate}

\section{Crystal data}

$\left(\mathrm{C}_{2} \mathrm{H}_{10} \mathrm{~N}_{2}\right)_{3}\left[\mathrm{Pr}_{2}\left(\mathrm{SO}_{4}\right)_{6}\right] \cdot 6 \mathrm{H}_{2} \mathrm{O}$

$M_{r}=1152.70$

Monoclinic, $P 2_{1} / c$

Hall symbol: -P $2 \mathrm{ybc}$

$a=6.6174(8) \AA$

$b=26.668(4) \AA$

$c=10.0264(13) \AA$

$\beta=104.446(15)^{\circ}$

$V=1713.4(4) \AA^{3}$

$Z=2$

\section{Data collection}

Stoe IPDS-II

diffractometer

Radiation source: fine-focus sealed tube

Graphite monochromator

$\omega$ and $\varphi$ scans

Absorption correction: multi-scan

( $X$-SHAPE and X-RED32; Stoe \& Cie, 2002)

$T_{\min }=0.491, T_{\max }=0.620$

\section{Refinement}

Refinement on $F^{2}$

Least-squares matrix: full

$R\left[F^{2}>2 \sigma\left(F^{2}\right)\right]=0.028$

$w R\left(F^{2}\right)=0.069$

$S=0.97$

3923 reflections

311 parameters

0 restraints
$F(000)=1148$

$D_{\mathrm{x}}=2.234 \mathrm{Mg} \mathrm{m}^{-3}$

Mo $K \alpha$ radiation, $\lambda=0.71073 \AA$

Cell parameters from 25 reflections

$\theta=20.0-24.3^{\circ}$

$\mu=3.29 \mathrm{~mm}^{-1}$

$T=295 \mathrm{~K}$

Parallelepiped, light-green

$0.22 \times 0.21 \times 0.20 \mathrm{~mm}$

14346 measured reflections

3922 independent reflections

3091 reflections with $I>2 \sigma(I)$

$R_{\text {int }}=0.044$

$\theta_{\max }=28.1^{\circ}, \theta_{\min }=2.6^{\circ}$

$h=-8 \rightarrow 8$

$k=-34 \rightarrow 35$

$l=-13 \rightarrow 13$

Primary atom site location: structure-invariant direct methods

Secondary atom site location: difference Fourier map

Hydrogen site location: inferred from neighbouring sites

All H-atom parameters refined

$w=1 /\left[\sigma^{2}\left(F_{\mathrm{o}}^{2}\right)+(0.0454 P)^{2}\right]$

where $P=\left(F_{\mathrm{o}}{ }^{2}+2 F_{\mathrm{c}}{ }^{2}\right) / 3$ 
$(\Delta / \sigma)_{\max }<0.001$

$\Delta \rho_{\max }=0.72{\mathrm{e} \AA^{-3}}^{-3}$

$\Delta \rho_{\min }=-1.08$ e $\AA^{-3}$
Extinction correction: SHELXL, $\mathrm{Fc}^{*}=\mathrm{kFc}\left[1+0.001 \times \mathrm{Fc}^{2} \lambda^{3} / \sin (2 \theta)\right]^{-1 / 4}$

Extinction coefficient: 0.0150 (6)

\section{Special details}

Experimental. A suitable single-crystal was carefully selected under a polarizing microscope and mounted in a glass capillary. The scattering intensities were collected on an imaging plate diffractometer (IPDS II, Stoe \& Cie) equipped with a fine focus sealed tube X-ray source (Mo K $\alpha, \lambda=0.71073 \AA$ ) operating at $50 \mathrm{kV}$ and $30 \mathrm{~mA}$. Intensity data for the title compound were collected at room temperature by $\omega$-scans in 180 frames $\left(0<\omega<180^{\circ} ; \varphi=0^{\circ}\right.$ and $90^{\circ}, \Delta \omega=2^{\circ}$, exposure time of $10 \mathrm{~min}$ ) in the $2 \Theta$ range 2.29 to $59.53^{\circ}$. Structure solution and refinement were carried out using the programs SIR97 (Altomare et al., 1999) and SHELXL97 (Sheldrick, 2008). The last cycles of refinement included atomic positions and anisotropic parameters for all atoms. The final difference maps were free of any chemically significant features. The refinement was based on $\mathrm{F}^{2}$ for ALL reflections.

Geometry. All e.s.d.'s (except the e.s.d. in the dihedral angle between two 1.s. planes) are estimated using the full covariance matrix. The cell e.s.d.'s are taken into account individually in the estimation of e.s.d.'s in distances, angles and torsion angles; correlations between e.s.d.'s in cell parameters are only used when they are defined by crystal symmetry. An approximate (isotropic) treatment of cell e.s.d.'s is used for estimating e.s.d.'s involving 1.s. planes.

Refinement. Refinement of $F^{2}$ against ALL reflections. The weighted $R$-factor $w R$ and goodness of fit $S$ are based on $F^{2}$, conventional $R$-factors $R$ are based on $F$, with $F$ set to zero for negative $F^{2}$. The threshold expression of $F^{2}>\sigma\left(F^{2}\right)$ is used only for calculating $R$-factors(gt) etc. and is not relevant to the choice of reflections for refinement. $R$-factors based on $F^{2}$ are statistically about twice as large as those based on $F$, and $R$ - factors based on ALL data will be even larger.

Fractional atomic coordinates and isotropic or equivalent isotropic displacement parameters $\left(\AA^{2}\right)$

\begin{tabular}{|c|c|c|c|c|}
\hline & $x$ & $y$ & $z$ & $U_{\text {iso }} * / U_{\text {eq }}$ \\
\hline $\operatorname{Pr}$ & $0.54622(3)$ & $0.177147(7)$ & 0.465046 (19) & $0.01075(8)$ \\
\hline $\mathrm{S} 1$ & $0.40656(17)$ & $0.06427(3)$ & $0.24648(10)$ & $0.0167(2)$ \\
\hline $\mathrm{S} 2$ & $0.52984(15)$ & $0.25100(3)$ & $0.21431(9)$ & $0.01307(18)$ \\
\hline S3 & $0.03251(16)$ & $0.15704(4)$ & $0.54283(9)$ & $0.0158(2)$ \\
\hline O11 & $0.1979(5)$ & $0.05421(12)$ & $0.1565(3)$ & $0.0283(7)$ \\
\hline O12 & $0.5682(6)$ & $0.05230(12)$ & $0.1764(3)$ & $0.0294(7)$ \\
\hline O13 & $0.4331(5)$ & $0.03414(11)$ & $0.3740(3)$ & $0.0269(7)$ \\
\hline O14 & $0.4203(5)$ & $0.11866(10)$ & $0.2836(3)$ & $0.0259(7)$ \\
\hline $\mathrm{O} 21$ & $0.5603(5)$ & $0.30594(11)$ & $0.2203(3)$ & $0.0205(6)$ \\
\hline $\mathrm{O} 22$ & $0.7151(5)$ & $0.22494(11)$ & $0.3007(3)$ & $0.0205(6)$ \\
\hline $\mathrm{O} 23$ & $0.3524(5)$ & $0.23557(10)$ & $0.2694(3)$ & $0.0183(6)$ \\
\hline $\mathrm{O} 24$ & $0.4972(5)$ & $0.23684(10)$ & $0.0667(3)$ & $0.0177(6)$ \\
\hline O31 & $0.1767(5)$ & $0.17700(11)$ & $0.4653(3)$ & $0.0222(6)$ \\
\hline $\mathrm{O} 32$ & $0.1426(6)$ & $0.12685(14)$ & $0.6597(4)$ & $0.0382(9)$ \\
\hline $\mathrm{O} 33$ & $-0.1327(5)$ & $0.12693(11)$ & $0.4480(3)$ & $0.0235(6)$ \\
\hline $\mathrm{O} 34$ & $-0.0807(5)$ & $0.19921(12)$ & $0.5890(3)$ & $0.0227(6)$ \\
\hline O1 & $0.5360(6)$ & $0.09619(12)$ & $0.5899(4)$ & $0.0298(8)$ \\
\hline H11 & $0.505(12)$ & $0.097(3)$ & $0.654(8)$ & $0.06(2)^{*}$ \\
\hline H12 & $0.501(9)$ & $0.074(2)$ & $0.539(6)$ & $0.030(15)^{*}$ \\
\hline $\mathrm{O} 2$ & $0.5314(10)$ & $0.1112(3)$ & $0.9028(7)$ & $0.100(3)$ \\
\hline $\mathrm{H} 21$ & $0.438(17)$ & $0.082(4)$ & $0.883(10)$ & $0.12(4)^{*}$ \\
\hline $\mathrm{H} 22$ & $0.495(12)$ & $0.138(3)$ & $0.877(8)$ & $0.06(2)^{*}$ \\
\hline $\mathrm{O} 3$ & $0.2361(7)$ & $0.03214(16)$ & $0.8914(4)$ & $0.0380(9)$ \\
\hline H31 & $0.220(11)$ & $0.033(3)$ & $0.975(8)$ & $0.056(19)^{*}$ \\
\hline H32 & $0.310(13)$ & 0.009 (3) & $0.888(8)$ & $0.07(3)^{*}$ \\
\hline
\end{tabular}




$\begin{array}{lllll}\text { N1 } & -0.0784(7) & 0.02857(16) & 0.6520(5) & 0.0283(8) \\ \text { H1A } & -0.090(11) & 0.060(3) & 0.623(7) & 0.06(2)^{*} \\ \text { H1B } & -0.005(11) & 0.032(3) & 0.738(7) & 0.053(19)^{*} \\ \text { H1C } & -0.204(14) & 0.008(3) & 0.646(9) & 0.08(3)^{*} \\ \text { C1 } & 0.0524(8) & 0.0008(2) & 0.5757(5) & 0.0300(10) \\ \text { H1D } & 0.082(10) & -0.034(3) & 0.615(6) & 0.048(17)^{*} \\ \text { H1E } & 0.173(11) & 0.014(2) & 0.595(6) & 0.045(18)^{*} \\ \text { N2 } & 0.0775(8) & 0.20727(16) & -0.1020(4) & 0.0257(8) \\ \text { H2A } & 0.183(11) & 0.216(2) & -0.057(7) & 0.040(18)^{*} \\ \text { H2B } & -0.011(12) & 0.230(3) & -0.113(7) & 0.06(2)^{*} \\ \text { H2C } & 0.086(10) & 0.199(2) & -0.192(7) & 0.042(16)^{*} \\ \text { C2 } & 0.0133(9) & 0.16060(18) & -0.0433(5) & 0.0278(10) \\ \text { H2D } & 0.115(10) & 0.136(2) & -0.031(6) & 0.044(17)^{*} \\ \text { H2E } & -0.095(13) & 0.144(3) & -0.107(8) & 0.08(3)^{*} \\ \text { N3 } & -0.1249(8) & 0.12597(16) & 0.1435(5) & 0.0279(9) \\ \text { H3A } & -0.245(11) & 0.119(2) & 0.094(6) & 0.039(17)^{*} \\ \text { H3B } & -0.023(12) & 0.103(3) & 0.153(7) & 0.06(2)^{*} \\ \text { H3C } & -0.126(11) & 0.129(3) & 0.230(8) & 0.05(2)^{*} \\ \text { C3 } & -0.0508(8) & 0.17187(17) & 0.0867(5) & 0.0257(9) \\ \text { H3D } & 0.054(10) & 0.184(2) & 0.148(6) & 0.031(15)^{*} \\ \text { H3E } & -0.145(9) & 0.197(2) & 0.085(6) & 0.035(15)^{*} \\ & & & & \end{array}$

Atomic displacement parameters $\left(\AA^{2}\right)$

\begin{tabular}{lllllll}
\hline & $U^{11}$ & $U^{22}$ & $U^{33}$ & $U^{12}$ & $U^{13}$ & $U^{23}$ \\
\hline Pr & $0.01069(13)$ & $0.01111(11)$ & $0.01082(11)$ & $0.00001(7)$ & $0.00336(7)$ & $-0.00053(8)$ \\
S1 & $0.0203(6)$ & $0.0136(4)$ & $0.0153(4)$ & $-0.0014(3)$ & $0.0026(3)$ & $-0.0027(3)$ \\
S2 & $0.0144(5)$ & $0.0144(4)$ & $0.0107(4)$ & $0.0007(3)$ & $0.0038(3)$ & $0.0022(3)$ \\
S3 & $0.0113(5)$ & $0.0182(4)$ & $0.0184(4)$ & $0.0002(3)$ & $0.0046(3)$ & $0.0041(4)$ \\
O11 & $0.0219(18)$ & $0.0306(17)$ & $0.0283(16)$ & $-0.0023(13)$ & $-0.0014(13)$ & $-0.0069(13)$ \\
O12 & $0.032(2)$ & $0.0285(17)$ & $0.0332(17)$ & $0.0017(13)$ & $0.0178(14)$ & $-0.0027(13)$ \\
O13 & $0.037(2)$ & $0.0216(15)$ & $0.0219(15)$ & $-0.0055(13)$ & $0.0063(13)$ & $0.0041(12)$ \\
O14 & $0.038(2)$ & $0.0132(14)$ & $0.0225(15)$ & $-0.0009(12)$ & $0.0005(13)$ & $-0.0048(11)$ \\
O21 & $0.0296(18)$ & $0.0156(13)$ & $0.0170(13)$ & $-0.0024(11)$ & $0.0070(12)$ & $-0.0003(10)$ \\
O22 & $0.0148(16)$ & $0.0265(15)$ & $0.0203(14)$ & $0.0032(11)$ & $0.0045(11)$ & $0.0095(11)$ \\
O23 & $0.0148(16)$ & $0.0227(14)$ & $0.0192(13)$ & $-0.0006(11)$ & $0.0075(11)$ & $0.0064(11)$ \\
O24 & $0.0223(16)$ & $0.0190(14)$ & $0.0128(13)$ & $0.0025(11)$ & $0.0064(11)$ & $0.0012(10)$ \\
O31 & $0.0144(16)$ & $0.0258(15)$ & $0.0290(15)$ & $-0.0014(11)$ & $0.0101(11)$ & $0.0065(12)$ \\
O32 & $0.0218(19)$ & $0.049(2)$ & $0.042(2)$ & $0.0036(15)$ & $0.0032(15)$ & $0.0301(17)$ \\
O33 & $0.0167(17)$ & $0.0166(14)$ & $0.0376(17)$ & $-0.0033(11)$ & $0.0076(12)$ & $-0.0072(12)$ \\
O34 & $0.0159(17)$ & $0.0282(16)$ & $0.0243(15)$ & $-0.0017(12)$ & $0.0057(12)$ & $-0.0115(12)$ \\
O1 & $0.050(2)$ & $0.0174(16)$ & $0.0246(17)$ & $-0.0035(14)$ & $0.0150(16)$ & $-0.0012(14)$ \\
O2 & $0.067(4)$ & $0.106(5)$ & $0.101(5)$ & $-0.043(4)$ & $-0.026(3)$ & $0.074(4)$ \\
O3 & $0.049(3)$ & $0.039(2)$ & $0.0219(17)$ & $0.0071(18)$ & $0.0004(15)$ & $-0.0071(15)$ \\
N1 & $0.030(2)$ & $0.023(2)$ & $0.033(2)$ & $-0.0027(16)$ & $0.0083(17)$ & $-0.0027(17)$ \\
C1 & $0.023(3)$ & $0.037(3)$ & $0.028(2)$ & $0.001(2)$ & $0.0030(19)$ & $-0.004(2)$ \\
N2 & $0.026(3)$ & $0.025(2)$ & $0.026(2)$ & $-0.0076(17)$ & $0.0056(17)$ & $0.0038(16)$ \\
C2 & $0.036(3)$ & $0.023(2)$ & $0.030(2)$ & $0.0009(19)$ & $0.020(2)$ & $0.0038(18)$ \\
& & & & & &
\end{tabular}


supporting information

\begin{tabular}{lllllll}
$\mathrm{N} 3$ & $0.026(3)$ & $0.033(2)$ & $0.028(2)$ & $-0.0022(17)$ & $0.0126(18)$ & $0.0050(17)$ \\
$\mathrm{C} 3$ & $0.028(3)$ & $0.022(2)$ & $0.030(2)$ & $-0.0035(18)$ & $0.0127(19)$ & $0.0000(18)$ \\
\hline
\end{tabular}

Geometric parameters $(\AA, \stackrel{o}{)}$

\begin{tabular}{|c|c|c|c|}
\hline $\mathrm{Pr}-\mathrm{O} 14$ & $2.383(3)$ & $\mathrm{O} 34-\mathrm{Pr}^{\mathrm{iv}}$ & $2.541(3)$ \\
\hline $\mathrm{Pr}-\mathrm{O} 31$ & $2.446(3)$ & $\mathrm{O} 1-\mathrm{H} 11$ & $0.72(8)$ \\
\hline $\mathrm{Pr}-\mathrm{O} 1$ & $2.505(3)$ & $\mathrm{O} 1-\mathrm{H} 12$ & $0.78(6)$ \\
\hline $\mathrm{Pr}-\mathrm{O} 34^{\mathrm{i}}$ & $2.541(3)$ & $\mathrm{O} 2-\mathrm{H} 21$ & $1.00(12)$ \\
\hline $\mathrm{Pr}-\mathrm{O} 22$ & $2.551(3)$ & $\mathrm{O} 2-\mathrm{H} 22$ & $0.77(8)$ \\
\hline $\mathrm{Pr}-\mathrm{O} 33^{\mathrm{i}}$ & $2.553(3)$ & $\mathrm{O} 3-\mathrm{H} 31$ & $0.87(7)$ \\
\hline $\mathrm{Pr}-\mathrm{O} 24^{\mathrm{ii}}$ & $2.564(3)$ & $\mathrm{O} 3-\mathrm{H} 32$ & $0.80(8)$ \\
\hline $\mathrm{Pr}-\mathrm{O} 21^{\mathrm{ii}}$ & $2.577(3)$ & $\mathrm{N} 1-\mathrm{C} 1$ & $1.487(6)$ \\
\hline $\mathrm{Pr}-\mathrm{O} 23$ & $2.582(3)$ & $\mathrm{N} 1-\mathrm{H} 1 \mathrm{~A}$ & $0.87(8)$ \\
\hline $\mathrm{Pr}-\mathrm{S} 3^{\mathrm{i}}$ & $3.1621(11)$ & $\mathrm{N} 1-\mathrm{H} 1 \mathrm{~B}$ & $0.88(7)$ \\
\hline $\mathrm{Pr}-\mathrm{S} 2^{\mathrm{ii}}$ & $3.1725(9)$ & $\mathrm{N} 1-\mathrm{H} 1 \mathrm{C}$ & $0.99(9)$ \\
\hline $\mathrm{Pr}-\mathrm{S} 2$ & $3.1741(9)$ & $\mathrm{C} 1-\mathrm{C} 1^{\mathrm{v}}$ & $1.504(9)$ \\
\hline $\mathrm{S} 1-\mathrm{O} 12$ & $1.454(3)$ & $\mathrm{C} 1-\mathrm{H} 1 \mathrm{D}$ & $1.01(7)$ \\
\hline $\mathrm{S} 1-\mathrm{O} 11$ & $1.473(3)$ & $\mathrm{C} 1-\mathrm{H} 1 \mathrm{E}$ & $0.84(7)$ \\
\hline $\mathrm{S} 1-\mathrm{O} 13$ & $1.483(3)$ & $\mathrm{N} 2-\mathrm{C} 2$ & $1.483(6)$ \\
\hline $\mathrm{S} 1-\mathrm{O} 14$ & $1.495(3)$ & $\mathrm{N} 2-\mathrm{H} 2 \mathrm{~A}$ & $0.76(7)$ \\
\hline $\mathrm{S} 2-\mathrm{O} 23$ & $1.475(3)$ & $\mathrm{N} 2-\mathrm{H} 2 \mathrm{~B}$ & $0.83(8)$ \\
\hline $\mathrm{S} 2-\mathrm{O} 21$ & $1.478(3)$ & $\mathrm{N} 2-\mathrm{H} 2 \mathrm{C}$ & $0.94(7)$ \\
\hline $\mathrm{S} 2-\mathrm{O} 22$ & $1.485(3)$ & $\mathrm{C} 2-\mathrm{C} 3$ & $1.499(7)$ \\
\hline $\mathrm{S} 2-\mathrm{O} 24$ & $1.490(3)$ & $\mathrm{C} 2-\mathrm{H} 2 \mathrm{D}$ & $0.92(7)$ \\
\hline $\mathrm{S} 3-\mathrm{O} 32$ & $1.458(3)$ & $\mathrm{C} 2-\mathrm{H} 2 \mathrm{E}$ & $0.94(8)$ \\
\hline $\mathrm{S} 3-\mathrm{O} 31$ & $1.472(3)$ & $\mathrm{N} 3-\mathrm{C} 3$ & $1.484(6)$ \\
\hline $\mathrm{S} 3-\mathrm{O} 34$ & $1.488(3)$ & $\mathrm{N} 3-\mathrm{H} 3 \mathrm{~A}$ & $0.85(7)$ \\
\hline $\mathrm{S} 3-\mathrm{O} 33$ & $1.492(3)$ & $\mathrm{N} 3-\mathrm{H} 3 \mathrm{~B}$ & $0.90(7)$ \\
\hline $\mathrm{O} 21-\mathrm{Pr}^{\mathrm{iii}}$ & $2.577(3)$ & $\mathrm{N} 3-\mathrm{H} 3 \mathrm{C}$ & $0.87(7)$ \\
\hline $\mathrm{O} 24-\mathrm{Pr}^{\mathrm{iii}}$ & $2.564(3)$ & $\mathrm{C} 3-\mathrm{H} 3 \mathrm{D}$ & $0.87(6)$ \\
\hline $\mathrm{O} 33-\operatorname{Pr}^{\mathrm{iv}}$ & $2.553(3)$ & $\mathrm{C} 3-\mathrm{H} 3 \mathrm{E}$ & $0.92(6)$ \\
\hline $\mathrm{O} 14-\mathrm{Pr}-\mathrm{O} 31$ & $80.82(11)$ & $\mathrm{O} 31-\mathrm{S} 3-\mathrm{O} 33$ & $109.05(18)$ \\
\hline $\mathrm{O} 14-\mathrm{Pr}-\mathrm{O} 1$ & $76.67(11)$ & $\mathrm{O} 34-\mathrm{S} 3-\mathrm{O} 33$ & $105.04(17)$ \\
\hline $\mathrm{O} 31-\mathrm{Pr}-\mathrm{O} 1$ & 81.17 (12) & $\mathrm{S} 1-\mathrm{O} 14-\mathrm{Pr}$ & $144.42(18)$ \\
\hline $\mathrm{O} 14-\mathrm{Pr}-\mathrm{O} 34^{\mathrm{i}}$ & $129.57(10)$ & $\mathrm{S} 2-\mathrm{O} 21-\mathrm{Pr}^{\mathrm{iii}}$ & 99.34 (13) \\
\hline $\mathrm{O} 31-\mathrm{Pr}-\mathrm{O} 34^{\mathrm{i}}$ & $148.16(10)$ & $\mathrm{S} 2-\mathrm{O} 22-\mathrm{Pr}$ & $100.34(14)$ \\
\hline $\mathrm{O} 1-\mathrm{Pr}-\mathrm{O} 34^{\mathrm{i}}$ & $95.70(12)$ & $\mathrm{S} 2-\mathrm{O} 23-\mathrm{Pr}$ & $99.30(14)$ \\
\hline $\mathrm{O} 14-\mathrm{Pr}-\mathrm{O} 22$ & $87.71(10)$ & $\mathrm{S} 2-\mathrm{O} 24-\mathrm{Pr}^{\mathrm{iii}}$ & $99.60(14)$ \\
\hline $\mathrm{O} 31-\mathrm{Pr}-\mathrm{O} 22$ & $126.92(9)$ & $\mathrm{S} 3-\mathrm{O} 31-\mathrm{Pr}$ & $141.33(18)$ \\
\hline $\mathrm{O} 1-\mathrm{Pr}-\mathrm{O} 22$ & $145.50(11)$ & $\mathrm{S} 3-\mathrm{O} 33-\mathrm{Pr}^{\mathrm{iv}}$ & 99.49 (14) \\
\hline $\mathrm{O} 34-\mathrm{i}-\mathrm{Pr} 22$ & $70.82(9)$ & $\mathrm{S} 3-\mathrm{O} 34-\mathrm{Pr}^{\mathrm{iv}}$ & $100.13(14)$ \\
\hline $\mathrm{O} 14-\mathrm{Pr}-\mathrm{O} 33^{\mathrm{i}}$ & $75.16(10)$ & $\mathrm{Pr}-\mathrm{O} 1-\mathrm{H} 11$ & $117(6)$ \\
\hline $\mathrm{O} 31-\mathrm{Pr}-\mathrm{O} 33^{\mathrm{i}}$ & $148.01(9)$ & $\mathrm{Pr}-\mathrm{O} 1-\mathrm{H} 12$ & $112(4)$ \\
\hline $\mathrm{O} 1-\mathrm{Pr}-\mathrm{O} 33^{\mathrm{i}}$ & $73.03(12)$ & $\mathrm{H} 11-\mathrm{O} 1-\mathrm{H} 12$ & $121(7)$ \\
\hline $\mathrm{O} 34^{\mathrm{i}}-\mathrm{Pr}-\mathrm{O} 33^{\mathrm{i}}$ & $55.31(9)$ & $\mathrm{H} 21-\mathrm{O} 2-\mathrm{H} 22$ & $122(8)$ \\
\hline $\mathrm{O} 22-\mathrm{Pr}-\mathrm{O} 33^{\mathrm{i}}$ & $73.30(10)$ & $\mathrm{H} 31-\mathrm{O} 3-\mathrm{H} 32$ & $107(7)$ \\
\hline
\end{tabular}




\begin{tabular}{|c|c|}
\hline $\mathrm{O} 14-\mathrm{Pr}-\mathrm{O} 24^{\mathrm{ii}}$ & $146.56(10)$ \\
\hline $\mathrm{O} 31-\mathrm{Pr}-\mathrm{O} 24^{\mathrm{ii}}$ & $77.02(10)$ \\
\hline $\mathrm{O} 1-\mathrm{Pr}-\mathrm{O} 24^{\mathrm{ii}}$ & $123.41(11)$ \\
\hline $\mathrm{O} 34^{\mathrm{i}}-\mathrm{Pr}-\mathrm{O} 24^{\mathrm{ii}}$ & $78.58(9)$ \\
\hline $\mathrm{O} 22-\mathrm{Pr}-\mathrm{O} 24^{\mathrm{ii}}$ & $85.94(9)$ \\
\hline $\mathrm{O} 33^{\mathrm{i}}-\mathrm{Pr}-\mathrm{O} 24^{\mathrm{ii}}$ & $133.31(9)$ \\
\hline $\mathrm{O} 14-\mathrm{Pr}-\mathrm{O} 21^{\mathrm{ii}}$ & $142.34(10)$ \\
\hline $\mathrm{O} 31-\mathrm{Pr}-\mathrm{O} 21^{\mathrm{ii}}$ & $77.75(10)$ \\
\hline $\mathrm{O} 1-\mathrm{Pr}-\mathrm{O} 21^{\mathrm{ii}}$ & $69.73(11)$ \\
\hline $\mathrm{O} 34^{\mathrm{i}}-\mathrm{Pr}-\mathrm{O} 21^{\mathrm{ii}}$ & $71.56(10)$ \\
\hline $\mathrm{O} 22-\mathrm{Pr}-\mathrm{O} 21^{\mathrm{ii}}$ & $129.86(10)$ \\
\hline $\mathrm{O} 33^{\mathrm{i}}-\mathrm{Pr}-\mathrm{O} 21^{\mathrm{ii}}$ & $109.50(10)$ \\
\hline $\mathrm{O} 24^{\mathrm{ii}}-\mathrm{Pr}-\mathrm{O} 21^{\mathrm{ii}}$ & $54.92(8)$ \\
\hline $\mathrm{O} 14-\mathrm{Pr}-\mathrm{O} 23$ & $78.58(10)$ \\
\hline $\mathrm{O} 31-\mathrm{Pr}-\mathrm{O} 23$ & $72.22(9)$ \\
\hline $\mathrm{O} 1-\mathrm{Pr}-\mathrm{O} 23$ & $146.05(12)$ \\
\hline $\mathrm{O} 34 \mathrm{i}-\mathrm{Pr}-\mathrm{O} 23$ & $118.03(10)$ \\
\hline $\mathrm{O} 22-\mathrm{Pr}-\mathrm{O} 23$ & $54.71(9)$ \\
\hline $\mathrm{O} 33^{\mathrm{i}}-\mathrm{Pr}-\mathrm{O} 23$ & $121.97(10)$ \\
\hline $\mathrm{O} 24^{\mathrm{ii}}-\mathrm{Pr}-\mathrm{O} 23$ & $70.99(9)$ \\
\hline $\mathrm{O} 21^{\mathrm{ii}}-\mathrm{Pr}-\mathrm{O} 23$ & $122.48(9)$ \\
\hline $\mathrm{O} 12-\mathrm{S} 1-\mathrm{O} 11$ & $110.7(2)$ \\
\hline $\mathrm{O} 12-\mathrm{S} 1-\mathrm{O} 13$ & $110.8(2)$ \\
\hline $\mathrm{O} 11-\mathrm{S} 1-\mathrm{O} 13$ & 108.69 (19) \\
\hline $\mathrm{O} 12-\mathrm{S} 1-\mathrm{O} 14$ & 109.09 (19) \\
\hline $\mathrm{O} 11-\mathrm{S} 1-\mathrm{O} 14$ & $108.60(19)$ \\
\hline $\mathrm{O} 13-\mathrm{S} 1-\mathrm{O} 14$ & $108.92(17)$ \\
\hline $\mathrm{O} 23-\mathrm{S} 2-\mathrm{O} 21$ & $111.99(17)$ \\
\hline $\mathrm{O} 23-\mathrm{S} 2-\mathrm{O} 22$ & $105.65(16)$ \\
\hline $\mathrm{O} 21-\mathrm{S} 2-\mathrm{O} 22$ & $110.84(18)$ \\
\hline $\mathrm{O} 23-\mathrm{S} 2-\mathrm{O} 24$ & $111.67(17)$ \\
\hline $\mathrm{O} 21-\mathrm{S} 2-\mathrm{O} 24$ & $106.03(16)$ \\
\hline $\mathrm{O} 22-\mathrm{S} 2-\mathrm{O} 24$ & $110.75(17)$ \\
\hline $\mathrm{O} 32-\mathrm{S} 3-\mathrm{O} 31$ & $111.4(2)$ \\
\hline $\mathrm{O} 32-\mathrm{S} 3-\mathrm{O} 34$ & $110.9(2)$ \\
\hline $\mathrm{O} 31-\mathrm{S} 3-\mathrm{O} 34$ & $109.50(18)$ \\
\hline $\mathrm{O} 32-\mathrm{S} 3-\mathrm{O} 33$ & $110.8(2)$ \\
\hline
\end{tabular}

\begin{tabular}{|c|c|}
\hline $\mathrm{C} 1-\mathrm{N} 1-\mathrm{H} 1 \mathrm{~A}$ & $108(5)$ \\
\hline $\mathrm{C} 1-\mathrm{N} 1-\mathrm{H} 1 \mathrm{~B}$ & $108(5)$ \\
\hline $\mathrm{H} 1 \mathrm{~A}-\mathrm{N} 1-\mathrm{H} 1 \mathrm{~B}$ & $102(6)$ \\
\hline $\mathrm{C} 1-\mathrm{N} 1-\mathrm{H} 1 \mathrm{C}$ & $106(5)$ \\
\hline $\mathrm{H} 1 \mathrm{~A}-\mathrm{N} 1-\mathrm{H} 1 \mathrm{C}$ & $120(7)$ \\
\hline $\mathrm{H} 1 \mathrm{~B}-\mathrm{N} 1-\mathrm{H} 1 \mathrm{C}$ & $111(6)$ \\
\hline $\mathrm{N} 1-\mathrm{C} 1-\mathrm{C} 1^{\mathrm{v}}$ & $110.7(5)$ \\
\hline $\mathrm{N} 1-\mathrm{C} 1-\mathrm{H} 1 \mathrm{D}$ & $110(4)$ \\
\hline $\mathrm{C} 1^{\mathrm{v}}-\mathrm{C} 1-\mathrm{H} 1 \mathrm{D}$ & $111(4)$ \\
\hline $\mathrm{N} 1-\mathrm{C} 1-\mathrm{H} 1 \mathrm{E}$ & $109(4)$ \\
\hline $\mathrm{C} 1^{\mathrm{v}}-\mathrm{C} 1-\mathrm{H} 1 \mathrm{E}$ & $114(4)$ \\
\hline $\mathrm{H} 1 \mathrm{D}-\mathrm{C} 1-\mathrm{H} 1 \mathrm{E}$ & $102(5)$ \\
\hline $\mathrm{C} 2-\mathrm{N} 2-\mathrm{H} 2 \mathrm{~A}$ & $109(5)$ \\
\hline $\mathrm{C} 2-\mathrm{N} 2-\mathrm{H} 2 \mathrm{~B}$ & $114(5)$ \\
\hline $\mathrm{H} 2 \mathrm{~A}-\mathrm{N} 2-\mathrm{H} 2 \mathrm{~B}$ & $112(7)$ \\
\hline $\mathrm{C} 2-\mathrm{N} 2-\mathrm{H} 2 \mathrm{C}$ & $106(4)$ \\
\hline $\mathrm{H} 2 \mathrm{~A}-\mathrm{N} 2-\mathrm{H} 2 \mathrm{C}$ & $112(6)$ \\
\hline $\mathrm{H} 2 \mathrm{~B}-\mathrm{N} 2-\mathrm{H} 2 \mathrm{C}$ & $104(6)$ \\
\hline $\mathrm{N} 2-\mathrm{C} 2-\mathrm{C} 3$ & $110.4(4)$ \\
\hline $\mathrm{N} 2-\mathrm{C} 2-\mathrm{H} 2 \mathrm{D}$ & $112(4)$ \\
\hline $\mathrm{C} 3-\mathrm{C} 2-\mathrm{H} 2 \mathrm{D}$ & $112(4)$ \\
\hline $\mathrm{N} 2-\mathrm{C} 2-\mathrm{H} 2 \mathrm{E}$ & $112(5)$ \\
\hline $\mathrm{C} 3-\mathrm{C} 2-\mathrm{H} 2 \mathrm{E}$ & $110(5)$ \\
\hline $\mathrm{H} 2 \mathrm{D}-\mathrm{C} 2-\mathrm{H} 2 \mathrm{E}$ & $100(6)$ \\
\hline $\mathrm{C} 3-\mathrm{N} 3-\mathrm{H} 3 \mathrm{~A}$ & $108(4)$ \\
\hline $\mathrm{C} 3-\mathrm{N} 3-\mathrm{H} 3 \mathrm{~B}$ & $107(5)$ \\
\hline $\mathrm{H} 3 \mathrm{~A}-\mathrm{N} 3-\mathrm{H} 3 \mathrm{~B}$ & $118(6)$ \\
\hline $\mathrm{C} 3-\mathrm{N} 3-\mathrm{H} 3 \mathrm{C}$ & $113(5)$ \\
\hline $\mathrm{H} 3 \mathrm{~A}-\mathrm{N} 3-\mathrm{H} 3 \mathrm{C}$ & $111(6)$ \\
\hline $\mathrm{H} 3 \mathrm{~B}-\mathrm{N} 3-\mathrm{H} 3 \mathrm{C}$ & $99(6)$ \\
\hline $\mathrm{N} 3-\mathrm{C} 3-\mathrm{C} 2$ & $111.2(4)$ \\
\hline $\mathrm{N} 3-\mathrm{C} 3-\mathrm{H} 3 \mathrm{D}$ & $109(4)$ \\
\hline $\mathrm{C} 2-\mathrm{C} 3-\mathrm{H} 3 \mathrm{D}$ & $110(4)$ \\
\hline $\mathrm{N} 3-\mathrm{C} 3-\mathrm{H} 3 \mathrm{E}$ & $109(4)$ \\
\hline $\mathrm{C} 2-\mathrm{C} 3-\mathrm{H} 3 \mathrm{E}$ & $118(4)$ \\
\hline $\mathrm{H} 3 \mathrm{D}-\mathrm{C} 3-\mathrm{H} 3 \mathrm{E}$ & $99(5)$ \\
\hline
\end{tabular}

Symmetry codes: (i) $x+1, y, z$; (ii) $x,-y+1 / 2, z+1 / 2$; (iii) $x,-y+1 / 2, z-1 / 2$; (iv) $x-1, y, z$; (v) $-x,-y,-z+1$.

Hydrogen-bond geometry $\left(\AA,{ }^{\circ}\right)$

\begin{tabular}{lllll}
\hline$D-\mathrm{H} \cdots A$ & $D-\mathrm{H}$ & $\mathrm{H} \cdots A$ & $D \cdots A$ & $D-\mathrm{H} \cdots A$ \\
\hline $\mathrm{O} 1-\mathrm{H} 11 \cdots \mathrm{O} 32$ & $0.72(8)$ & $2.53(8)$ & $2.974(6)$ & $121(7)$ \\
$\mathrm{O} 1-\mathrm{H} 12 \cdots \mathrm{O} 13$ & $0.78(6)$ & $1.92(6)$ & $2.674(5)$ & $162(6)$ \\
$\mathrm{O} 2-\mathrm{H} 21 \cdots \mathrm{O} 3$ & $1.00(12)$ & $1.89(12)$ & $2.858(7)$ & $163(9)$ \\
$\mathrm{O} 2-\mathrm{H} 22 \cdots \mathrm{O} 21^{\mathrm{ii}}$ & $0.77(8)$ & $2.29(8)$ & $2.905(6)$ & $137(7)$ \\
$\mathrm{O} 3-\mathrm{H} 31 \cdots \mathrm{O} 11^{\mathrm{vi}}$ & $0.87(7)$ & $1.95(8)$ & $2.795(5)$ & $165(7)$
\end{tabular}


supporting information

\begin{tabular}{|c|c|c|c|c|}
\hline $\mathrm{O} 3-\mathrm{H} 32 \cdots \mathrm{O} 12^{\mathrm{vii}}$ & $0.80(8)$ & $2.00(8)$ & $2.766(5)$ & $162(8)$ \\
\hline $\mathrm{N} 1-\mathrm{H} 1 A \cdots \mathrm{O} 33$ & $0.87(8)$ & $2.48(8)$ & $3.291(5)$ & $155(6)$ \\
\hline $\mathrm{N} 1-\mathrm{H} 1 B \cdots \mathrm{O} 3$ & $0.88(7)$ & $1.92(7)$ & $2.758(6)$ & $158(6)$ \\
\hline $\mathrm{N} 1-\mathrm{H} 1 C^{\cdots} \mathrm{O} 13^{\mathrm{v}}$ & $0.99(9)$ & $1.85(9)$ & $2.841(6)$ & $176(7)$ \\
\hline $\mathrm{N} 2-\mathrm{H} 2 A \cdots \mathrm{O} 24$ & $0.76(7)$ & $2.21(7)$ & $2.976(5)$ & $177(7)$ \\
\hline $\mathrm{N} 2-\mathrm{H} 2 B \cdots \mathrm{O} 22^{\text {viii }}$ & $0.83(8)$ & $2.17(8)$ & $2.967(6)$ & $162(7)$ \\
\hline $\mathrm{N} 2-\mathrm{H} 2 C \cdots \mathrm{O} 34^{\mathrm{ix}}$ & $0.94(7)$ & $2.20(6)$ & $3.020(5)$ & $146(5)$ \\
\hline $\mathrm{N} 3-\mathrm{H} 3 A \cdots \mathrm{O} 2^{\mathrm{x}}$ & $0.85(7)$ & $2.12(7)$ & $2.901(8)$ & $153(6)$ \\
\hline $\mathrm{N} 3-\mathrm{H} 3 B \cdots \mathrm{O} 11$ & $0.90(7)$ & $1.95(8)$ & $2.847(6)$ & $175(6)$ \\
\hline $\mathrm{N} 3-\mathrm{H} 3 C^{-\cdots} \mathrm{O} 33$ & $0.87(7)$ & $2.20(7)$ & $3.066(5)$ & $173(6)$ \\
\hline
\end{tabular}

Symmetry codes: (ii) $x,-y+1 / 2, z+1 / 2$; (v) $-x,-y,-z+1$; (vi) $x, y, z+1$; (vii) $-x+1,-y,-z+1$; (viii) $x-1,-y+1 / 2, z-1 / 2$; (ix) $x, y, z-1$; (x) $x-1, y, z-1$. 